\title{
SPECTRAL FEATURE ANALYSIS FOR QUANTITATIVE ESTIMATION OF CYA- NOBACTERIA CHLOROPHYLL-A
}

\author{
Yi Lin a,b, Zhanglin Ye ${ }^{a, b}$, Yugan Zhang a,b, Jie Yu ${ }^{a, b}$ \\ ${ }^{a}$ College of Surveying and Geoinformatics, Tongji University, Shanghai 200092, China-(linyi, \\ 2011_jieyu)@tongji.edu.cn,a443561491@outlook.com,zhangyuguan@outlook.com \\ ${ }^{\mathrm{b}}$ Research Center of Remote Sensing and Spatial Information Technology, Tongji University, Shanghai 200092, China-(linyi, \\ 2011_jieyu)@tongji.edu.cn,a443561491@outlook.com,zhangyuguan@outlook.com
}

KEY WORDS: Chlorophyll- $a$, Spectral analysis, Multivariate statistical analysis, Vegetation indices, Broad band, Narrow band

\begin{abstract}
:
In recent years, lake eutrophication caused a large of Cyanobacteria bloom which not only brought serious ecological disaster but also restricted the sustainable development of regional economy in our country. Chlorophyll- $a$ is a very important environmental factor to monitor water quality, especially for lake eutrophication. Remote sensed technique has been widely utilized in estimating the concentration of chlorophyll-a by different kind of vegetation indices and monitoring its distribution in lakes, rivers or along coastline. For each vegetation index, its quantitative estimation accuracy for different satellite data might change since there might be a discrepancy of spectral resolution and channel center between different satellites. The purpose of this paper is to analyze the spectral feature of chlorophyll- $a$ with hyperspectral data (totally 651 bands) and use the result to choose the optimal band combination for different satellites. The analysis method developed here in this study could be useful to recognize and monitor cyanobacteria bloom automatically and accurately.
\end{abstract}

In our experiment, the reflectance (from $350 \mathrm{~nm}$ to1000nm) of wild cyanobacteria in different consistency (from 0 to $1362.11 \mathrm{ug} / \mathrm{L}$ ) and the corresponding chlorophyll-a concentration were measured simultaneously. Two kinds of hyperspectral vegetation indices were applied in this study: simple ratio (SR) and narrow band normalized difference vegetation index (NDVI), both of which consists of any two bands in the entire 651 narrow bands. Then multivariate statistical analysis was used to construct the linear, power and exponential models. After analyzing the correlation between chlorophyll- $a$ and single band reflectance, SR, NDVI respectively, the optimal spectral index for quantitative estimation of cyanobacteria chlorophyll-a, as well as corresponding central wavelength and band width were extracted. Results show that: Under the condition of water disturbance, SR and NDVI are both suitable for quantitative estimation of chlorophyll- $a$, and more effective than the traditional single band model; the best regression models for SR, NDVI with chlorophyll- $a$ are linear and power, respectively. Under the condition without water disturbance, the single band model works the best. For the SR index, there are two optimal band combinations, which is comprised of infrared (700nm-900nm) and blue-green range $(450 \mathrm{~nm}-550 \mathrm{~nm})$, infrared and red range $(600 \mathrm{~nm}-650 \mathrm{~nm})$ respectively, with band width between $45 \mathrm{~nm}$ to $125 \mathrm{~nm}$. For NDVI, the optimal band combination includes the range from $750 \mathrm{~nm}$ to $900 \mathrm{~nm}$ and from $700 \mathrm{~nm}$ to $750 \mathrm{~nm}$, with band width less than 30nm.For single band model, band center located between $733 \mathrm{~nm}-935 \mathrm{~nm}$, and its width mustn't exceed the interval where band center located in.

This study proved, as for SR or NDVI, the centers and widths are crucial factors for quantitative estimating chlorophyll-a. As for remote sensor, proper spectrum channel could not only improve the accuracy of recognizing cyanobacteria bloom, but reduce the redundancy of hyperspectral data. Those results will provide better reference for designing the suitable spectrum channel of custom- 
ized sensors for cyanobacteria bloom monitoring at a low altitude. In other words, this study is also the basic research for developing the real-time remote sensing monitoring system with high time and high spatial resolution

\section{INTRODUCTION}

In recent years, lake eutrophication caused a large of Cyanobacteria bloom which not only brought serious ecological disaster but also restricted the sustainable development of regional economy in our country, it has become a pretty serious environmental problem. In order to reduce the water pollution caused by cyanobacterial bloom and ensure the safety of drinking water, many satellite data have been used to study the spatial distribution (Matthew, 2012), and the biomass quantitative retrieval (LIU Tangyou, 2002; Heng Lyu, 2013) for water bloom. Biological risk caused by cyanobacteria bloom was evaluated, while cyanobacteria bloom was monitored and predicted, and water quality early warning system was established (Gower, 1994; Adam, 2000; FENG Jiangfan et al, 2009). These satellite data can be classified into wide band multispectral data and narrow band hyperspectral data according to the spectral resolution, hyperspectral data generally comes from field spectrometer and aerial image. According to the previous researches, narrow band can provide more significant information about the physical characteristics for quantitative research.

Chlorophyll- $a$ is a kind of important environment factor which can be utilized to evaluate water quality, nutrient load and pollution level. In the past decades, many vegetation indices were used to quantify the biological variation of aquatic plants, for example Simple Ratio (SR) is often used to estimate the biological variation, such as cyanobacteria, leucocyan etc. Normalized Difference Vegetation Index (NDVI) is an effective index that can monitor vegetation and ecotope (Ekstran, 1992; MA Ronghua et al, 2005; Sachidananda Mishra, 2013; Wesley J.Moses, 2012; Changchun Huang, 2014). In spite of the same vegetation indices, there also would be discrepancy in quantitative estimation precision, since the band location and width of satellite spectral channel are different. As for spectral index, the center location and band width have a lot of impact on the precision of quantitative estimation.

The main purpose of this paper is to analyze the quantified ability for chlorophyll- $a$ corresponding to different vegetation indices calculated from hyperspectral data. Optimal spectral indices and what band wavelength and band center were determined, which are suitable for quantitative estimation for the chlorophyll-a of cyanobacteria. So that hyperspectral data redundancy can be reduced and the accuracy can be improved while quantitative estimating cyanobacteria. The results significantly provide basic research to monitoring and early warning on cyanobacteria bloom using multispectral data or hyperspectral data.

\section{DATA AND METHOD}

\subsection{Algae selection}

Cyanobacterial blooms are common phenomena which are caused by eutrophication (KONG Fanxiang et al, 2005). Cyanobacteria could come into bloom when the natural condition becomes suitable for cyanobacteria. Microcystic aeruginosa is the one of the most common cyanobacteria species, which can lead to bloom easily, in China. The majority of previous researches usually used the Microcystic aeruginosa, captuered in laboratory, whose feature is different from the wild Microcystic aeruginosa in micromorphologic, macromorphologic and cybotactic state. However, it could rarely gather together and generate bloom. Thus in our experiment the control experiments were conducted by using wild Microcystic aeruginosa in Tai Lake.

\subsection{Control experiment}

The FieldSpecFR spectrometer manufactured by American ASD Company has been applied in this research. The spectrum consist of visible and near-infrared (VNIR), short wave infrared 1 (SWIR1) and short wave infrared 2 (SWIR2). After interpolating the spectral channels, spectral data was obtained, of which the spectral resolution is $1 \mathrm{~nm}$. The VNIR range $(350 \mathrm{~nm}-1000 \mathrm{~nm})$ was used in our research.

Two control experiments were conducted to get spectral data on 2nd October 2014 and 3rd October 2014 respectively. In the first experiment, two plastic boxes $(0.45 \mathrm{~m} * 0.35 \mathrm{~m} * 0.3 \mathrm{~m})$ were used as containers. Then one black plastic bag was placed inside of each box to remove the influence of projection light. The absolute reflectance in each container is less than $1 \%$ 
(400nm-800nm). The probe of spectrometer was vertically placed over the container, looking straight down at a height of $0.3 \mathrm{~m}$ from water surface. Surface detection range was a circle with a radius of $0.067 \mathrm{~m}$. During the experiment, firstly enough water was poured into the container (water is pure without suspended solids). Then a certain amount of Microcystic aerugino$s a$ was added drop by drop, meanwhile stirred uniformly. Each time after adding Microcystic aeruginosa, three samples were collected to obtain the concentration of chlorophyll-a, and measured spectral data three times (under the condition of water disturbance). Five minutes later, when the water came back calm and Microcystic aeruginosa distributed uniformly on water surface, spectral data was measured three times again (under the condition of water stationary). These two containers were utilized alternately during the experiment. Totally, 16 groups of different concentration spectral data were collected. The concentration of chlorophyll-a increased at the speed of 1.3 times, ranging from $0-1190.46 \mu \mathrm{g} / \mathrm{L}$. To obtain more reference data, in the second experiment, the same procedure was performed. A group of sub-experiments were under the condition of disturbance, and the range of chlorophyll-a concentration was $15.31 \mu \mathrm{g} / \mathrm{L}-450.9 \mu \mathrm{g} / \mathrm{L}$ (12 gradients). Then a group of sub-experiment was under the condition of water stationary, the range of chlorophyll-a concentration was $143.64 \mu \mathrm{g} / \mathrm{L}$ $-1362.11 \mu \mathrm{g} / \mathrm{L}$ (9 gradients).

The spectral reflectance is calculated by the following equation:

$$
\rho_{T}=\frac{L_{T}}{L_{r}} \times \kappa
$$

Where $\rho_{T}=$ spectral reflectance

$$
\begin{aligned}
& \mathrm{L}_{\mathrm{T}}=\text { target radiance } \\
& \mathrm{Lr}=\text { reference plate radiance } \\
& \kappa=\text { reference plate reflectance }
\end{aligned}
$$

In our research, a standard gray plate was used as the reference plate, whose reflectance is $30 \%$. The quantitative estimation ability of narrow band vegetation models, broad band vegetation indices models and chlorophyll-a were compared respectively, based on which the optimal spectral width can be extracted. Meanwhile Landsat ETM+ bands were selected as the representative of broad bands. Spectral response function was used, and spectral response values of Landsat ETM+'s first 4 bands were simulated by ASD spectral reflectance (Steven et al,
2003). Before applying convolution to ASD spectral reflectance, smoothing processing was not used while obtaining the broad band spectral data.

\subsection{Multivariate statistical analysis}

Multivariate statistical analysis is generally applied in the study of ocean color remote sensing (Andrew Clive Banks, 2012). Original spectrum, simple ratio (SR) and normalized difference vegetation index (NDVI) (Table 1) were chosen as independent variables, and the concentration of Microcystic aeruginosa chlorophyll- $a$ was regarded as the dependent variable. Pearson product-moment correlation coefficient $(r)$ was calculated to describe the correlation between dependent variable (y) and independent variable $(\mathrm{x})$ :

$$
r=\frac{\sum_{i=1}^{n}\left(x_{i}-\bar{x}\right)\left(\mathrm{y}_{i}-\bar{y}\right)}{\sqrt{\sum_{i=1}^{n}\left(x_{i}-\bar{x}\right)^{2}} \sqrt{\sum_{i=1}^{n}\left(\mathrm{y}_{i}-\bar{y}\right)^{2}}}
$$

Where $\bar{x}=$ average of independent variables

$\bar{y}=$ average of dependent variables

$\mathrm{n}=$ the number of dependent variables/independent variables

Then intervals in which $\mathrm{x}$, y located with high degree of correlation were chosen to construct a linear prediction model.

Actually, there might be non-linear relationship between original spectrum, SR, NDVI and chlorophyll-a respectively, so another two non-linear prediction models were used: power and exponential in addition:

According to the result of determination coefficient $\left(\mathrm{R}^{2}\right)$ :

$$
R^{2}=\frac{\left(\sum_{i=1}^{n} x_{i} y_{i}\right)^{2}}{\left(\sum_{i=1}^{n} x_{i}^{2}\right)\left(\sum_{i=1}^{n} y_{i}^{2}\right)}
$$

The optimal prediction model would be chosen and the precision of predicted value would be evaluated with root mean square error (RMSE) and mean relative error (MRE). Data ob- 
tained in the first experiment were applied as forecast samples, then the data from second experiment were regarded as test samples.

\section{RESULTS AND ANALYSIS}

Spectral reflectance of water and Microcystic aeruginosa with different concentration were obtained by control experiments, under the condition of water disturbance and stationary. Spectral reflectance of bands whose wavelength located between $350 \mathrm{~nm}-1000 \mathrm{~nm}$ was used. Meanwhile two different narrow band vegetation indices and corresponding broad band vegetation indices were calculated (Table 1). Narrow band vegetation indices consisted of any two bands in the entire 651 narrow bands while broad band vegetation indices were the combinations of Landsat ETM+ NIR and RED bands.

These specific spectral indices were:

- Broad band ETM SR and NDVI indices;

- Narrow SR and NDVI indices: any two bands in the entire 651 bands.

\begin{tabular}{ccc}
\hline Vegetation indices & Definition \\
\hline SR & Broad band & $S R=\frac{N I R}{R E D}$ \\
& Narrow band & $S R_{i j}=\frac{\rho_{j}}{\rho_{i}}$ \\
NDVI & Broad band & $N D V I=\frac{N I R-\mathrm{RED}}{N I R+\mathrm{RED}}$ \\
& Narrow band & $N D V I_{i j}=\frac{\rho_{j}-\rho_{i}}{\rho_{j}+\rho_{i}}$ \\
\hline
\end{tabular}

Table 1: Spectral vegetation indices used in this paper

$$
\begin{aligned}
\text { Where NIR } & =\text { Landsat } \mathrm{ETM}+\mathrm{B} 4 \text { bands } \\
\mathrm{RED} & =\text { Landsat } \mathrm{ETM}+\mathrm{B} 3 \text { bands. }
\end{aligned}
$$

\subsection{Correlation of single band reflectance and Microcystic} aeruginosa chlorophyll-a

Figure 1 shows the correlation coefficient (r) between single band reflectance and chlorophyll-a under the condition of water disturbance and stationary.

The trend of $\mathrm{r}$ is very close to the Microcystic aeruginosa spec- tral reflectance. In the band intervals in which $\mathrm{r}$ decreased and increased quickly, Microcystic aeruginosa spectral reflectance has the similar change. In this area, reflectance is very sensitive to chlorophyll-a concentration. The location of 3 minimum reflectance is corresponding to 3 reflection troughs of Microcystic aeruginosa spectrum; the location of 3 maximum is corresponding to Microcystic aeruginosa spectral green range peak, secondary band peak, red edge respectively.

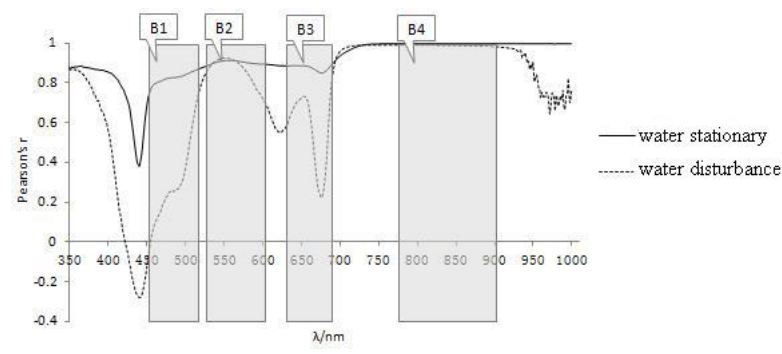

Figure 1 Correlation coefficient between Microcystic aerugino$s a$ spectral reflectance and chlorophyll-a concentration

3.2 Correlation of broad band vegetation indices and $\mathrm{Mi}$ crocystic aeruginosa chlorophyll-a

Under the condition of water disturbance and stationary, correlation coefficient $(r>0.9)$ of Microcystic aeruginosa chlorophyll-a with band 2, band 4 are both very high. At band 1 and band 3 , correlation coefficient $(r<0.5)$ is low under the condition of water disturbance. On the contrary, $r$ is more than 0.85 under the condition of water stationary (Figure 2).

The independent variables include single band reflectance from $733 \mathrm{~nm}$ to $794 \mathrm{~nm}$, ETM's band 4 reflectance whose central band is $835 \mathrm{~nm}$, the SR and NDVI consisted of ETM's band $3 \& 4$ and ETM's band $3 \&$ band 4 central bands. With these variables, linear prediction model of chlorophyll- $a$ was constructed. The precision was evaluated by calculating determination coefficient

\begin{tabular}{|c|c|c|c|c|}
\hline \multirow{2}{*}{$\begin{array}{l}\text { Independent } \\
\text { variable }\end{array}$} & \multirow[b]{2}{*}{ condition } & \multicolumn{2}{|l|}{ prediction } & \multirow{2}{*}{$\begin{array}{c}\text { Test } \\
\text { RMSE }\end{array}$} \\
\hline & & function & $\mathrm{R}^{2}$ & \\
\hline 799nm & $\mathrm{D}$ & $y=8010.8 x-112.55$ & 0.9850 & 84.45 \\
\hline $794 \mathrm{~nm}$ & $S$ & $y=2355.8 x-13.65$ & 0.9903 & 34.15 \\
\hline \multirow{2}{*}{$835 \mathrm{~nm}$} & $\mathrm{D}$ & $y=10090 x-116.83$ & 0.9805 & 85.43 \\
\hline & S & $y=2369.2 x-2.2073$ & 0.9912 & 31.76 \\
\hline
\end{tabular}
and RMSE (Table 2). 


\begin{tabular}{ccccl}
\hline ETM_B4 & $\mathrm{D}$ & $\mathrm{y}=9818 \mathrm{x}-116.6$ & 0.9812 & 87.23 \\
& $\mathrm{~S}$ & $\mathrm{y}=2373 \mathrm{x}-3.518$ & 0.9912 & 32.34 \\
ETM_SR & $\mathrm{D}$ & $\mathrm{y}=341.4 \mathrm{x}-233.5$ & $\mathbf{0 . 9 9 2 8}$ & $\mathbf{3 0 . 9}$ \\
& $\mathrm{S}$ & $\mathrm{y}=124.8 \mathrm{x}-98.87$ & 0.9921 & 53.82 \\
C_SR & $\mathrm{D}$ & $\mathrm{y}=364.19 \mathrm{x}-241.6$ & $\mathbf{0 . 9 9 5 6}$ & $\mathbf{3 1 . 9 6}$ \\
& $\mathrm{S}$ & $\mathrm{y}=127.98 \mathrm{x}-98.01$ & 0.9921 & 53.46 \\
ETM_NDVI & $\mathrm{D}$ & $\mathrm{y}=1276.4 \mathrm{x}+125.7$ & 0.8693 & 113.8 \\
& $\mathrm{~S}$ & $\mathrm{y}=985.4 \mathrm{x}-68.04$ & 0.7193 & 133.7 \\
C_NDVI & $\mathrm{D}$ & $\mathrm{y}=1293.5 \mathrm{x}+149.92$ & 0.8776 & 111.6 \\
& $\mathrm{~S}$ & $\mathrm{y}=983.1 \mathrm{x}-56.508$ & 0.7258 & 133 \\
\hline
\end{tabular}

Table 2: linear model

ETM_SR and ETM_NDVI were used to represent broad band indices and C_SR and C_NDVI to narrow band indices. Water disturbance and stationary was abbreviated as $\mathrm{D}$ and $\mathrm{S}$ respectively. The relation between chlorophyll-a real value $\mathrm{Y}$ and predicted value $\mathrm{y}$ is:

$$
y=a * Y+b
$$

$$
\begin{array}{r}
\text { Where } \mathrm{a}=\text { gain } \\
\mathrm{b}=\text { bias }
\end{array}
$$

The RMSE is regarded as a scale used to compare each model's predictive ability. By comparing RMSE, under the condition of water disturbance, the predictive precision of SR model is higher, even though the $\mathrm{R}^{2}$ of single band model and SR model are both very high. Hence there is almost little discrepancy between narrow SR model and broad band SR model. Under the condition of water stationary, SR model's predictive precision is lower than that single band model's, where RMSE of SR model is 1.7 times higher than that of single band model. The predictive ability of ETM+'s band 4 model is close to which of $794 \mathrm{~nm}$ model.

\subsection{Correlation of narrow band vegetation indices and $\mathrm{Mi}$ - crocystic aeruginosa chlorophyll-a}

Narrow band SR and NDVI indices consist of any two bands in the entire 651 narrow bands $(651 * 651=423801)$ were calculated. All narrow band SR and NDVI were chose as independent variable and chlorophyll-a is as dependent variable to construct linear regression model. $\mathrm{R}^{2}$ was calculated and shown in Figure 2.The abscissa axis $\lambda_{1}$ and vertical axis $\lambda_{2}$ are $\rho_{\mathrm{j}}$ band and $\rho_{\mathrm{i}}$ band respectively, which were applied to SR and NDVI indices. The results of SR's linear regression models under the condition of water disturbance and stationary are shown in Figures 3 (A and B). While the results of NDVI's linear regression models under the condition of water disturbance and stationary are shown in Figures 3 (C and D).

According to the results above, in each isogram, 6 groups of SR and NDVI indices were extracted where $\mathrm{R}^{2}$ is the biggest, as well as corresponding band center $\lambda_{1}, \lambda_{2}$ and band width $\Delta \lambda_{1}$, $\Delta \lambda_{2}$ respectively. The area where $R^{2}>0.97$ was divided into two parts $\left(R^{2}>0.99\right.$ and $\left.0.97<R^{2}<0.99\right)$. In the part where $R^{2}>0.99,4$ rectangular regions can be extracted. They were SR indices SR1/2/3/6 under condition of water disturbance.
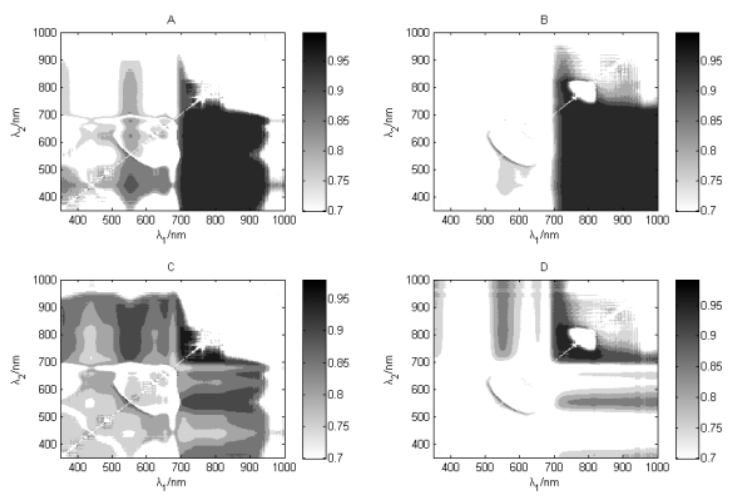

Figure $2 \mathrm{R}^{2}$ 's isogram of narrow band $\mathrm{SR}$, NDVI and chlorophyll-a

Strong linear correlations has been shown between these 6 indices and chlorophyll-a. Estimation ability of all SR indices that meet the following equation to chlorophyll- $a$ is similar.

$$
\mathrm{SR} 1=\frac{\rho_{j}\left(\lambda_{2} \pm \Delta_{2}\right)}{\rho_{i}\left(\lambda_{1} \pm \Delta_{1}\right)}
$$

Where $\Delta_{1}=\left(0-\Delta \lambda_{1} / 2\right) \mathrm{nm}$

$$
\Delta_{2}=\left(0-\Delta \lambda_{2} / 2\right) \mathrm{nm}
$$

There were totally 24 different results for six indices in this experiment (two types, two conditions). The most optimal narrow band width was found in the NDVI index under the condition of water stationary $\left(\Delta \lambda_{1}=8 \mathrm{~nm}, \Delta \lambda_{2}=4 \mathrm{~nm}\right)$; while the most optimal broad band width was found in the SR5 index under the 
condition of water disturbance $\left(\Delta \lambda_{1}=362 \mathrm{~nm}, \Delta \lambda_{2}=227 \mathrm{~nm}\right)$.

\subsection{Non-linear correlation of vegetation indices and chloro-} phyll-a

Figure 2 illustrates the linear correlation of SR, NDVI indices and Microcystic aeruginosa chlorophyll-a. However, sometimes there may be non-linear correlation between vegetation indices and chlorophyll-a. In our research, power model is the optimal non-linear model. 6 groups of optimal linear models' band center $\left(\lambda_{1}\right.$ and $\left.\lambda_{2}\right)$ and band width $\left(\Delta \lambda_{1}\right.$ and $\left.\Delta \lambda_{2}\right)$ were applied to calculate non-linear models. $\mathrm{R}^{2}$ and RMSE (Table 3) were calculated by optimal linear models and power models.

As shown in Table 3, for SR indices, SR1/2/3 linear models are better than those power models. While SR4/5/6 power models are better than those linear models under the condition of disturbance. Only SR4 power model performed better than those linear models under the condition with disturbance. For NDVI, under the condition of disturbance, estimation precision of power models is superior to their linear models. Under the condition of stationary, NDVI 1/2/3/5 linear models performed better than their power models. NDVI 4/6 and ETM_NDVI power models performed better than their linear models.

After comparing SR and NDVI, the indices' quantitative estimation precision of SR 1/2/3/4/6 indices and NDVI 5/6 can perform pretty well under the condition of water disturbance. SR indices and NDVI indices' quantitative estimation precision to chlorophyll- $a$ are similar, which both performed worse than their single band models.

\begin{tabular}{cccccc}
\hline & & \multicolumn{2}{c}{ Linear model } & \multicolumn{2}{c}{ Power model } \\
\hline condition & index & $\mathrm{R}^{2}$ & RMSE & $\mathrm{R}^{2}$ & RMSE \\
\hline \multirow{6}{*}{ disturbance } & SR1 & 0.995 & 31.76 & 0.9883 & 39.24 \\
& SR2 & 0.9971 & 31.46 & 0.9899 & 35.66 \\
& SR3 & 0.9947 & 36.04 & 0.9879 & 36.61 \\
& SR4 & 0.9631 & 69.44 & 0.9951 & 27.89 \\
& SR5 & 0.9582 & 135.3 & 0.9171 & 76.04 \\
& SR6 & 0.9916 & 44.17 & 0.9873 & 36.56 \\
& ETM_SR & 0.9952 & 30.9 & 0.9878 & 39.52 \\
\hline \multirow{6}{*}{ stationary } & SR1 & 0.9934 & 47.19 & 0.9949 & 49.02 \\
& SR2 & 0.9927 & 46.55 & 0.9947 & 50 \\
& SR3 & 0.9928 & 47.61 & 0.9946 & 52.13 \\
& SR4 & 0.9782 & 55.33 & 0.9952 & 47.88 \\
\hline
\end{tabular}

\begin{tabular}{cccccl}
\hline \multirow{6}{*}{ SR5 } & 0.9789 & 76.57 & 0.9049 & 123.1 \\
& SR6 & 0.9729 & 67.83 & 0.9191 & 112.1 \\
ETM_SR & 0.9921 & 54.02 & 0.9948 & 58.98 \\
\hline \multirow{4}{*}{ disturbance } & NDVI1 & 0.963 & 85.66 & 0.9753 & 49.37 \\
& NDVI2 & 0.9641 & 100.4 & 0.9563 & 59.08 \\
& NDVI3 & 0.9587 & 97.91 & 0.9659 & 55.5 \\
& NDVI4 & 0.9548 & 83.03 & 0.9794 & 50.04 \\
& NDVI5 & 0.9248 & 115.1 & 0.9966 & 29.46 \\
& NDVI6 & 0.9068 & 109.1 & 0.9948 & 33.3 \\
& ETM_NDVI & 0.8593 & 113.8 & 0.9936 & 32.47 \\
\hline \multirow{5}{*}{ stationary } & NDVI1 & 0.9879 & 72.5 & 0.9134 & 118.2 \\
& NDVI2 & 0.9848 & 64.26 & 0.9403 & 102 \\
& NDVI3 & 0.9639 & 73.97 & 0.9674 & 89.08 \\
& NDVI4 & 0.9236 & 92.74 & 0.9907 & 51.97 \\
& NDVI5 & 0.9516 & 79.68 & 0.9763 & 89.61 \\
& NDVI6 & 0.8609 & 106.1 & 0.9863 & 45.14 \\
& ETM_NDVI & 0.7193 & 133.7 & 0.9694 & 57.45 \\
\hline & & & & &
\end{tabular}

Table 3: 6 optimal linear models and non-linear models

\subsection{Distribution of optimal bands}

For the above 6 groups of indices, the optimal 4 groups were chosen to calculate the distribution of corresponding band center and band width (shown in Figure $4 \& 5$ ).

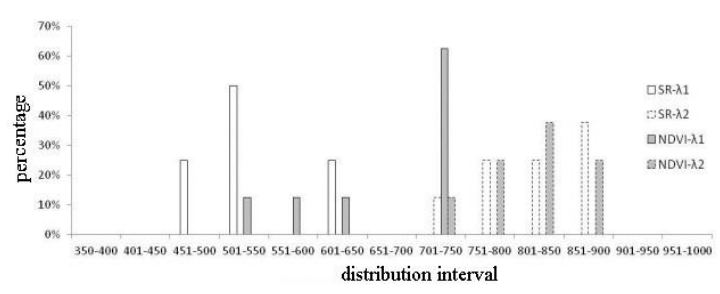

Figure 3: Distribution of SR and NDVI indices' band center
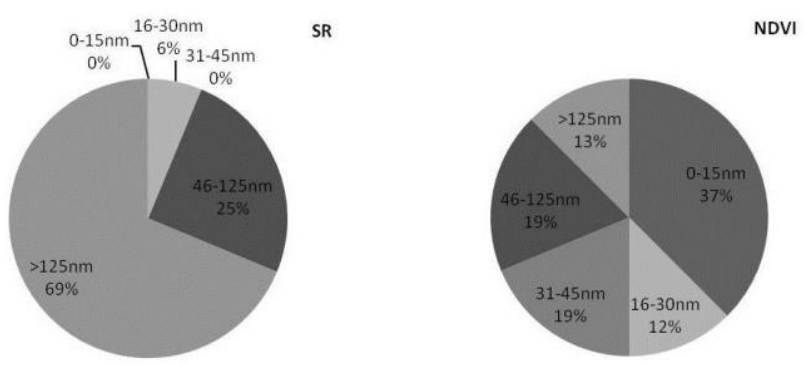

Figure 4: Distribution of SR and NDVI indices' band width

As shown in Figure 3 and 4, for SR indices, the most optimal 
band centers of $\rho_{\mathrm{i}}$ are between 450nm-550nm and $600 \mathrm{~nm}-650 \mathrm{~nm}$. And the most optimal band center of $\rho_{\mathrm{j}}(\lambda 1, \mathrm{NIR})$ locates in the range of $700 \mathrm{~nm}-900 \mathrm{~nm}$. The most optimal band widths locate between $46 \mathrm{~nm}-125 \mathrm{~nm}$ and more than $125 \mathrm{~nm}$. For NDVI, most $\rho_{\mathrm{i}}$ locate in the range from $700 \mathrm{~nm}$ to $750 \mathrm{~nm}$, and most $\rho_{\mathrm{j}}$ are between 750nm-900nm. Obviously, for SR indices, broad bands take an absolute advantage for quantitative estimating Microcystic aeruginosa chlorophyll-a. For NDVI, the narrower bands have, the better quantitative ability is.

\section{CONCLUSIONS}

By performing two control experiments, the correlation between chlorophyll- $a$ and single band reflectance, SR, NDVI were analyzed respectively. Linear and non-linear regression models were constructed. Then various spectral indices, which were suitable for quantitative estimation of Microcystic aeruginosa chlorophyll-a, their central band wavelength and band width were obtained, which are summarized herewith:

(1) Selecting spectral indices: Under the condition of water disturbance, SR and NDVI indices are both suitable for quantitative estimation of chlorophyll-a, which both performed better than those single band models. Linear model was suitable for SR indices, and power model was suitable for NDVI, under the condition of water stationary, single band models can estimate the concentration of chlorophyll-a pretty well, while SR and NDVI indices performed worse.

(2) Selecting band centers and width of spectral indices: For the SR index, there are two optimal band combinations, which is comprised of infrared (700nm-900nm) and blue-green range(450nm-550nm), infrared and red range (600nm-650nm) respectively, with band width between $45 \mathrm{~nm}$ to $125 \mathrm{~nm}$. For NDVI, the optimal band combination includes the range from $750 \mathrm{~nm}$ to $900 \mathrm{~nm}$ and from $700 \mathrm{~nm}$ to $750 \mathrm{~nm}$, with band width less than $30 \mathrm{~nm}$.For single band model, band center located between $733 \mathrm{~nm}-935 \mathrm{~nm}$, and its width mustn't exceed the interval where band center located in.

Our experiments analyzed the quantified ability between $\mathrm{Mi}$ crocystic aeruginosa chlorophyll-a and different vegetation indices under the disturbance and stationary conditions. The following two aspects could be improved: 1) Besides Microcystic aeruginosa, there is much suspended matter, chromophoric dissolved organic matter and so on which can influence the spectrum in lakes; 2) Atmospheric influence was ignored during simulating Landsat ETM data. So our results should be verified in the lake water.

\section{ACKNOWLEDGEMENTS}

Data were collected on the roof of Liren Building in FuDan University, we wish to express our gratitude to this. In addition, we wish to give a special thanks to Dr. Mingzhi QU from Queen's University, who provided much assistance in practices and theoretical guidance.

\section{REFERENCES}

FENG Jiangfan, TENG Xuewei, ZHANG Hong, XU Jie. 2006. Forewarning System of Blue Algae for Taihu Lake Based on GIS, 29(9):60-64.

HU Wen, WU Wenyu, KONG Qingxin, XUN Shangpei, WANG Yulan. 2002. Research on Estimating the Concentrations of Chlorophyll-a in Chaohu Lake Using FY-1C/CAVHRR Data, 25(1):124-128.

KONG FanXiang, GAO Guang. 2005. Hypothesis on cyanobacteria bloom-forming mechanism in large shallow eutrophic lakes, 25(3):589-595.

LIU TangYou, KUANG DingBo, YIN Qiu. 2002. The Spectrum experiments of Algae and Studies On Retrieval Quantitative Information From Its Spectra, 21(3): 213-217.

MA Ronghua, KONG Fanxiang, DUAN Hongtao. 2008. Spatio-temporal distribution of cyanobacteria blooms based on satellite imageries inLake Taihu, China, 20(6): 687-694.

Adams CM, Mulkey D, Hodges A, MilonJW . 2000. Development of an economic impact assessment methodology for occurrence of red tide. SP 00-12. Inst Food AgricSci, Food Resource Econ Dept, Univ Florida, Gainesville, Florida, 58 pp.

Changchun Huang, Jun Zou, Yunmei Li et al. 2014. Assessment of NIR-red algorithms for observation of chlorophyll-a in highly turbid inland waters in China. ISPRS Journal of Photogrammetry and Remote Sensing, 93:29-39.

Christopher D. Elvidge and Zhikang Chen. 1995. Comparison of Broad-Band and Narrow-Band Red and Near-Infrared Vege- 
tation Indices. Remote Sensing of Environment, 54:38-48.

Daniela Gurlin, Anatoly A. Gitelson, Wesley J.Moses. 2011. Remote estimation of chl-a concentration in turbid productive waters - Return to a simple two-band NIR-red model. Remote Sensing of Environment, 115: 3479- 3490.

Andrew Clive Banks, Pascal Prunet, Julien Chimot et al, A satellite ocean color observation operator system for eutrophication assessment in coastal waters, Journal of Marine Systems, Volume 94, Supplement, June 2012, Pages S2-S15, ISSN 0924-7963.

Ekstrand, S., 1992. Landsat TM based quantification of chlorophyll-a during algae blooms in coastal. Int. J. Remote Sens. 10, 1913-1926.

Donald C. Rundquist, Luoheng Han, John F. Schalles et al. 1996. Remote Measurement of Algal Chlorophyll in Surface Waters: The Case for the First Derivative of Reflectance Near 690 nm. Photogrammetric Engineering \& Remote Sensing,62:195-200.

EssayasKabaa, William Philpot, TammoSteenhuis. 2014. Evaluating suitability of MODIS-Terraimages for reproducing historicsediment concentrations in water bodies: Lake Tana, Ethiopia. International Journal of Applied Earth Observation and Geoinformation. 26: 286-297.

Gilerson, A.A., Gitelson, A.A., Zhou, J., Gurlin, D., Moses, W.J., Ioannou, I., Ahmed, S.A., 2010. Algorithms for remote estimation of chlorophyll-a in coastal and inland waters using red and near-infrared bands. Optics Express 18, 24109-24125.

Giltelson A., Kondratev K. 1991. Peak near 700nm in reflectance spectrum of productive waters and its application for remote monitoring of water quality. Transactions Doklady of the USSR Academy of Sciences: Earth Science Section,306:1-4.

GOWER, J.F.R., 1994. Red tide monitoring using AVHRR HRPT imagery from a local receiver. Remote Sensing of Environment, 48, pp. 309-318.

HengLyu, QiaoWang, ChuanqingWuet al.2013. Retrieval of phycocyanin concentration from remote-sensing reflectance using a semi-analytic model in eutrophic lakes. Ecological Informatics $18: 178-187$.

Matthews, M., Bernard, S., \& Robertson, L. 2012. An algorithm for detecting trophic status (chlorophyll-a), cyanobacterial-dominance, surface scums and floating vegetation in inland and coastal waters. Remote Sensing of Environment, 124, $637-652$.

Huete A, Didan K, Miura T et al. 2002. Overview of the radiometric and biophysical performance of the MODIS vegetation indices. Remote Sensing of Environment, 83:195-213.

Luoheng Han and Karen J. Jordan. 2005. Estimating and mapping chlorophyll-a concentration in Pensacola Bay, Florida using Landsat ETM+ data. International Journal of Remote Sensing,26(23):5245-5254.

Wei Shi, Junwu Tang. 2011. Water property monitoring and assessment for China's inland Lake Taihu from MODIS-Aqua measurements. Remote Sensing of Environment 115: 841-854.

Robert K. Vincenta,XiaomingQina,R. Michael L. McKay et al. 2004. Phycocyanin detection from LANDSAT TM data for mapping cyanobacterial blooms in Lake Erie. Remote Sensing of Environment,89: $381-392$

SachidanandaMishra , Deepak R. Mishra. 2012. Normalized difference chlorophyll index: A novel model for remote estimation of chlorophyll-a concentration in turbid productive waters. Remote Sensing of Environment, 117:394-406.

Sachidananda Mishra, Deepak R. Mishra, Zhongping Lee et al.2013. Quantifying cyanobacterial phycocyanin concentration in turbid productive waters:A quasi-analytical approach. Remote Sensing of Environment, 133:141-151.

Steven, M., Malthus, T., Baret, F., Xu, H., \& Chopping, M. 2003. Intercalibration of vegetation indices from different sensor systems. Remote Sensing of Environment, 88(4), 412-422.

Y. Chao Rodríguez, A. el Anjoumi, J. A. Domínguez Gómez et al. 2014. Using Landsat image time series to study a small water body in Northern Spain. Environ Monit Assess, 186:3511-3522.

Yacobi, Y.Z. Gitelson, A. Mayo, M. 1995, Remote sensing of chlorophyll in Lake Kinneret using high spectra resolution radiometer and Landsat TM: spectral features of reflectance and algorithm development. Journal of Plankton Research, 17, pp. 2155-2173. 